\title{
LE TRAVAIL DU PROFESSEUR DÉBUTANT DE FLE : LE RÔLE DES ORIENTATIONS DANS SON ACTIVITÉ
}

Fernanda SOARES

Mariana Casemiro BARIONI²

RÉSUMÉ : Cet article a pour but de présenter une analyse d'un extrait d'une Instruction au Sosie réalisée avec une professeure débutante de Français Langue Étrangère (FLE). Basée sur les Sciences du Travail, plus spécifiquement l'Ergonomie de l'Activité (AMIGUES, 2002 ; 2004 ; 2012 ; RIA, 2005 ; SAUJAT, 2004 ; AMIGUES ; FÉLIX ; ESPINASSY, 2014), sur la Clinique de l'Activité (CLOT, 2001 ; 2006) et sur le cadre théorique de l'Interactionnisme Socio-discursif (BRONCKART, 2003), cette étude vise à découvrir dans et à travers les textes d'Instruction au Sosie : quelles sont les représentations construites sur les orientations pour le travail du professeur débutant ? À partir de ces orientations, comment ce professeur organise son travail ?

MOTS-CLÉS : Instruction au Sosie, Ergonomie de l'Activité, Clinique de l'Activité, Interactionnisme Socio-discursif, Orientations pour le travail.

RESUMO : Este artigo tem como objetivo apresentar uma análise de um trecho de uma Instrução ao Sósia realizada com uma professora iniciante
1 Université de São Paulo - Faculté de Philosophie, Lettres et Sciences Humaines, Brésil. Étudiante en Master. fernandasoares@usp.br

2 Université de São Paulo -Faculté de Philosophie, Lettres et Sciences Humaines, Brésil. Étudiante en Master. mariana.barioni@usp.br 
de Francês Língua Estrangeira (FLE). Baseado em estudos das Ciências do Trabalho, mais precisamente na Ergonomia da Atividade (AMIGUES, 2002 ; 2004 ; 2012 ; RIA, 2005 ; SAUJAT, 2004 ; AMIGUES ; FÉLIX ; ESPINASSY, 2014), na Clínica da Atividade (CLOT, 2001 ; 2006) e no quadro teórico do Interacionismo Sociodiscursivo (BRONCKART, 2003), este estudo visa a descobrir nos e pelos textos de Instrução ao Sósia: quais são as representações construídas nos e através dos textos de Instrução ao Sósia sobre as orientações para o trabalho do professor iniciante? A partir dessas orientações, como o professor organiza seu trabalho?

PALAVRAS-CHAVE: Instrução ao Sósia, Ergonomia da Atividade, Clínica da Atividade, Interacionismo Sóciodiscursivo, Orientações para o trabalho. 


\section{Introduction}

Ce travail est inséré dans le cadre de nos recherches de master qui cherchent à étudier le travail du professeur débutant de français langue étrangère (FLE) à travers l'analyse des textes produits par les professeurs en leurs situations de travail.

De manière à contribuer aux études sur le travail du professeur de langues étrangères, surtout à celui des professeurs débutants, cette recherche a pour but de présenter une analyse d'un extrait d'un texte produit en situation de travail par une professeure débutante de FLE, où nous cherchons à réfléchir sur la présence des orientations dans son travail, leur importance et leur rôle dans l'activité de cette professeure.

L'objet de notre analyse est l'extrait que nous avons choisi ${ }^{3}$ de la transcription du texte d'instruction d'un entretien d'« Instruction au Sosie ». Il s'agit d'une méthode indirecte d'investigation utilisée par des chercheurs de la Clinique de l'Activité (CLOT, 2001; 2006) qui a pour but de créer une situation qui rend possible le développement professionnel du travailleur à travers une procédure d'entretien.

Le cadre théorique et méthodologique qui sous-tend cette étude est celui des Sciences du Travail, plus spécifiquement l'Ergonomie de l'Activité (AMIGUES, 2002 ; 2004 ; 2012 ; RIA, 2005 ; SAUJAT, 2004 ; AMIGUES ; FÉLIX ; ESPINASSY, 2014) et la Clinique de l'Activité (CLOT, 2001; 2006 ; FAÏTA, 2004), qui fournissent des moyens pour comprendre les situations vécues et les dilemmes rencontrés par les professeurs débutants en situation de travail. On s'est également basées sur les études de l'Interactionnisme Socio-discursif (BRONCKART, 2003), utilisé pour le traitement des données et aussi pour aider la compréhension du travail enseignant et ses plusieurs aspects.

Pour atteindre l'objectif de cette étude, nous cherchons à répondre aux questions suivantes :

a. Quelles sont les représentations construites dans et à travers les textes d'Instruction au Sosie sur les orientations pour le travail du professeur débutant?

b. À partir de ces orientations, comment ce professeur organise-t-il son travail ?

3 L'extrait choisi de l'Instruction au Sosie étudié ici se trouve en pièce jointe. 


\section{Cadre théorique}

Les bases théoriques et méthodologiques qui orientent notre article se fondent sur les études de l'Ergonomie de l'Activité (AMIGUES, 2002 ; 2004 ; 2012 ; RIA, 2005 ; SAUJAT, 2004 ; AMIGUES ; FÉLIX ; ESPINASSY, 2014) et de la Clinique de l'Activité (CLOT, 2001; 2006 ; FAÏTA, 2004) qui développent des concepts fondamentaux pour la compréhension du travail, l'une des façons de l'agir humain (LOUSADA, 2006). On considère, dans cette perspective, l'enseignement comme un travail (AMIGUES, 2003). Notre cadre théorique est aussi composé des propositions de l'Interactionnisme Socio-discursif, courant de l'Interactionnisme Social (VIGOTSKI, 1984 ; 2009) à travers les recherches développées par Bronckart (2003) et d'autres chercheurs appartenant au même cadre théorique (MACHADO, 2004 ; LOUSADA, 2006 ; 2011 ; MACHADO, LOUSADA, FERREIRA, 2011) qui cherchent à étudier l'agir humain, surtout l'agir langagier. L'Interactionnisme Socio-discursif (dorénavant ISD) propose un modèle d'analyse de textes que nous allons présenter par la suite et qui a été adopté dans cette investigation pour le traitement des données.

L'Ergonomie de l'Activité et la Clinique de l'Activité font partie de ce qu'on appelle les Sciences du Travail, dont la psychologie du travail, la psychopathologie du travail et d'autres courants théoriques. Elles se préoccupent des situations de travail, de leurs possibilités de transformation et de la subjectivité au milieu de travail. Ces préoccupations sont très attachées à l'Interactionnisme Social, ce qui justifie leur adoption à côté des idées de l'ISD. Selon ces perspectives théoriques, on ne peut pas comprendre le travail, plus spécifiquement ici le travail enseignant, indépendamment des prescriptions, des dimensions collectives qui l'organisent (AMIGUES, 2012) et des " résultats de l'activité de travail sur le propre travailleur » (LOUSADA, 2006 :72). Dans ce cadre théorique, on a de différentes situations de travail étudiées et la contribution de plusieurs concepts sur le travail. Ici, on en abordera deux qui sont importants pour cette étude : la distinction parmi le travail prescrit, réalisé et réel et la méthode d'instruction au Sosie.

Le travail prescrit est tout ce qui est prescrit, recommandé par les organisations de travail et ce qui doit être fait, accompli. Ce sont les textes, oraux ou écrits, qui préfigurent le travail, qui le précèdent dans sa réalisation sans forcément être prescriptifs. Le travail prescrit est la tâche donnée au travailleur, tandis que le travail réalisé est « l'activité effectivement réalisé » (LOUSADA, 2006 :75). Cepen- 
dant, il y a une distance inévitable entre le travail prescrit et le travail réalisé. Vygotski a dit que "l'homme est plein à chaque minute de possibilités non réalisées » (1994a apud CLOT, 2001). Ce qui a été fait, la possibilité réalisée, « est gouverné par les conflits entre activités rivales éliminées qui auraient pu, sans doute à d'autres coûts, réaliser la même tâche » (CLOT, 2001). Ce qui est réalisé est une petite partie de tout ce qui est possible ; c'est l'ensemble entier des possibilités, celles qui sont réalisées et aussi celles qui ne sont pas réalisées, que Clot propose d'appeler de «travail réel » (2001). Ce qui nous intéresse dans cet article est le travail réel, ce qui est visible et invisible du travail enseignant (AMIGUES ; FÉLIX ; ESPINASSY, 2014) vu que c'est dans cette catégorie qu'on trouve de l'espace pour les émotions, les inhibitions et les intentions (CLOT, 2006). Les possibilités mises de côté, celles qui n'ont pas été réalisées, ne sont pas accessibles directement, mais à partir des traces qui doivent être construites à partir de celles qui ont été réalisées.

L'observation de l'action, du travail réalisé, n'est pas suffisante pour rendre compte de la compréhension du travail réel, de tous les mécanismes et possibilités qui y sont rapportées ; pourtant elle peut constituer la trappe d'accès pour remonter à l'activité, au travail réel, à ce qui ne se voit pas : «ce qui ne se voit pas, l'activité, explique ce qui se voit, l'action » (AMIGUES ; FÉLIX ; ESPINASSY, 2014). Puisque "l'activité ne se touche pas du doigt »(HUBAULT, 1996 apud AMIGUES ; FÉLIX ; ESPINASSY, 2014) on doit employer des méthodes indirectes d'investigation (VYGOTSKI 1994a; 1994b; 1999 apud CLOT, 2001) pour accéder à l'activité inobservable, au réel de l'activité.

Les méthodes indirectes servent à saisir l'expérience professionnelle : elles permettent une reprise sans répétition de l'activité (CLOT, 2001) et leur but c'est de faire en sorte qu'une expérience vécue devienne un moyen qui permet de vivre une autre expérience. Une façon de le faire c'est l'inversion de rôles, qui permet, selon Lousada (2006 :79) que les sujets observés dans leurs situations de travail observent leurs propres activités, ce qui rend possible la prise de conscience de son activité par le travailleur, « un contact social avec soi-même » (VYGOTSKI, 1994a apud Clot, 2001).

Développée par Odonne dans les années 70 à la Fiat et reprise par Clot (2001) dans le cadre de recherche de la Psychologie du Travail à la Clinique de l'Activité du Conservatoire National des Arts et Métier (CNAM) à Paris, France, l'Instruction au Sosie (IS) est une méthode indirecte qui a pour but de créer une situation qui rend possible le développement professionnel du travailleur. Dans cette 
procédure, le chercheur se place à la position de sosie du sujet de recherche, celui qui doit donner au sosie des instructions pour qu'il (le sosie) puisse réaliser ses activités de travail sans que personne ne se rende compte du changement. À partir de ce procédé, il est possible de mettre en scène les détails d'une activité à être réalisée. Cet entretien est enregistré sur audio.

À partir de l'IS on obtient deux textes: le texte d'instruction et le texte de commentaire. Le texte d'instruction est la transcription de l'entretien, quand le sujet de recherche donne au sosie des instructions pour qu'il réalise ses activités de travail de façon que personne ne se rende compte du changement de rôles. Ensuite, le texte d'instruction est rendu au sujet de recherche pour qu'il enregistre ses impressions sur le contenu fourni/construit pendant les orientations données au sosie ; de cette façon, on a le texte commentaire. Nous partons de l'idée qu'il faut écouter la voix du travailleur pour comprendre comment son travail se configure à partir des textes produits lui-même.

Cet échange de rôles (sujet de recherche/sosie) fait surgir des spécificités de la situation de travail : des conflits, des empêchements et des difficultés pour la réalisation de l'activité. De cette manière, on croit que c'est dans et à travers les textes dans lesquels le travailleur commente/décrit/aborde son propre travail que nous pouvons accéder aux représentations (BRONCKART, 2003) des conflits vécus et des obstacles de l'agir de ce professionnel.

L'instruction au sosie est une méthode indirecte qui marche, pour le travailleur, comme un catalyseur de la réflexion sur son travail. Avec elle, le travailleur découvre lui-même les détails de son travail en pouvant voir son " travail en miettes ». Cette procédure vise à une transformation indirecte du travail des sujets « grâce à un déplacement de leurs activités dans un nouveau contexte » (CLOT, 2001).

Après avoir présenté le cadre théorique de la Clinique de l'Activité et de l'Ergonomie de l'Activité et deux notions fondamentales de ces perspectives pour cet article, on abordera par la suite l'Interactionnisme Socio-discursif.

L'ISD vise à montrer le rôle fondateur du langage et du fonctionnement de l'activité discursive dans le développement humain (BRONCKART, 2003). Dans cette perspective, les deux plus grandes unités d'analyse sont les actions et les textes. Ceux-ci peuvent être définis comme des produits empiriques des actions langagières, une unité communicative globale. Les actions sont des séquences organisées de comportement, isolées dans le flux continu de l'activité d'un 
groupe, qui peuvent être attribuées à un agent particulier (BRONCKART, 2003) et qui deviennent concrètes dans les textes. Ainsi, la seule manière d'analyser les actions est à travers les textes où elles deviennent concrètes, et pour cela l'ISD propose un modèle d'organisation interne des textes qui cherche à l'analyse des conditions de fonctionnement effectif des textes.

Selon ce modèle d'analyse, on commence l'étude du texte par les représentations sociales sur le monde physique et socio-subjectif où les producteurs du texte sont insérés (MACHADO, 2002). Ensuite, nous repérons l'organisation du texte qui est considérée comme un feuilleté textuel composé par trois strates superposés : l'infrastructure générale, les mécanismes de textualisation et les mécanismes énonciatifs ${ }^{4}$.

En ayant les mêmes apports théoriques et en s'appuyant sur Vygotski (1984; 2009) pour les questions d'ordre psychologiques, la Clinique de l'Activité, l'Ergonomie de l'Activité et l'ISD nous fournissent des sources pour comprendre, à travers l'analyse des textes, les représentations construites sur les orientations pour le travail du professeur et l'organisation du travail à partir des orientations.

\section{Méthodologie}

Nous présentons la méthodologie de notre étude organisée en quatre étapes, à savoir :

i) le contexte ; ii) les participants ; iii) la production des données et iv) l'analyse des données.

\section{i) Le contexte}

Cette étude appartient au cadre des recherches de master des deux auteures de cet article, développées à l'Université de São Paulo. Ces recherches visent à étudier le travail du professeur débutant de français langue étrangère (FLE). Ainsi, on y utilise les données qui ont été produites dans des contextes différents : tandis qu'une des participantes (Melissa) a comme contexte les Cours extra-universitaires de l'Université de São Paulo, l'autre (Fabrícia) a comme contexte une école de FLE privée dans une ville à l'intérieur de l'État de São Paulo. La différence de contextes et de façons de travailler est considérée, selon notre perspective, comme des aspects positifs, vu que c'est cela qui peut provoquer de la transformation chez les travailleurs et par conséquent dans leurs situations de travail.
4 Pour plus d'informations, voir Bronckart (2003). 


\section{ii) Les participants}

Le présent article a trois participants englobés directement dans le corpus analysé: Fabrícia, Melissa et Fábia. Fabrícia et Melissa sont des étudiantes en master et professeures débutantes de FLE. Fábia est professeure de FLE plus expérimentée que Fabrícia et Melissa et étudiante en doctorat. Pendant l'Instruction au Sosie (dont nous parlerons par la suite) Fabrícia et Melissa ont les rôles d'instructrice et Fábia est leur sosie. Elles ont une relation d'amitié ce qui justifie l'emploi $\mathrm{du} \ll \mathrm{tu}$ » lors de la procédure.

\section{iii) La production des données}

Nous avons réalisé la première étape de l'Instruction au Sosie (celle qui produit le texte d'instruction) le 30 septembre 2013 dans une salle du bâtiment de la Faculté de Philosophie, Lettres et Sciences Humaines de l'Université de São Paulo à São Paulo, Brésil. Nous avons réalisée l'Instruction au Sosie dans un petit collectif : deux instructrices (Fabrícia et Melissa) et le sosie, Fábia. On l’a organisée de la façon suivante : d'abord Fabrícia a donné les instructions de remplacement au sosie et Melissa a tout observé ; après, Melissa l'a fait et Fabrícia a tout observé ; ensuite, Fábia - pas en tant que sosie mais en tant que chercheuse - a ouvert un débat où les instructrices devraient discuter de la pratique de la collègue, ce qui a été remarqué de différent, d'intéressant ou d'autres commentaires. Ainsi, on a obtenu l'enregistrement audio de l'entretien. Les auteures ont fait la transcription de l'audio en utilisant les normes de transcription proposées par le projet NURC/SP (PRETI, 1999) et on a eu le texte d'instruction.

Fabrícia et Melissa ont lu le texte d'instruction et ont produit, chacune, un texte commentaire sur ce qu'elles ont pu remarquer et ce à quoi elles ont pu réfléchir et à partir du texte d'instruction. Le 27 novembre 2013 les trois participantes se sont donné rendez-vous à l'Université de São Paulo pour discuter des textes commentaires de chacune et faire un débat. Cela a été enregistré sur audio aussi et on l'a appelé de "retour de l'Instruction au Sosie ». Cet enregistrement audio a aussi été transcrit selon les normes de transcription déjà citées.

Pour cette investigation, les deux auteures ont choisi d'aborder ici un extrait qui contient des aspects capables de démontrer les représentations construites sur les orientations pour le travail enseignant dans et à travers les textes d'Instruction au Sosie. 


\section{iv) l'analyse des données}

Pour analyser l'extrait sélectionné nous utiliserons le modèle d'analyse de textes proposé par l'ISD ${ }^{5}$. Ainsi, l'extrait sera analysé selon les niveaux suivants :

- Le contexte de production physique et social.

- L'infrastructure globale : le plan global des contenus thématiques, les types de discours et les séquences.

- Les mécanismes de textualisation : la cohésion verbale, la cohésion nominale et la connexion.

- Les mécanismes énonciatifs : les modalisations et l’insertion de voix.

Ensuite, on l'analysera à partir des questions du travail abordées par la Clinique de l'Activité et l'Ergonomie que nous avons mentionnées dans le cadre théorique.

\section{Analyses}

Notre premier plan d'analyse de l'extrait choisi de l'IS réalisée est le contexte de production, qui englobe le contexte physique de production (un émetteur, un récepteur, un lieu de production et un moment de production) et le contexte socio-subjectif de production (un énonciateur, un destinataire, un lieu social et un but). Comme nous l'avons déjà affirmé, on analyse le texte selon le modèle d'analyse de textes proposé dans le cadre de l'ISD (BRONCKART, 2003).

En ce qui concerne le contexte physique, l'Instruction au Sosie réalisée par la sosie (Fábia) et par les instructrices (Melissa et Fabrícia), a été produite le 30 septembre 2013 dans une salle du bâtiment de la Faculté de Philosophie, Lettres et Sciences Humaines de l’Université de São Paulo au Brésil (comme on l'a déjà signalé dans la méthodologie). Pendant l'IS, les trois participantes ont décidé de parler en français parce que le français a le statut de langue de travail pour les trois, puisqu'elles sont professeurs de français langue étrangère (FLE).

Pour mieux comprendre le contexte de production du texte étudié ici, nous analyserons son contexte socio-subjectif en se centrant sur les participantes Fábia et Fabrícia, qui sont les sujets actifs ${ }^{6}$ de cette partie du texte, mais on considère toujours la présence de Melissa.

Fabrícia et Melissa sont professeures débutantes de FLE et étu-
5 Pour en connaître plus, voir Bronckart (2003).
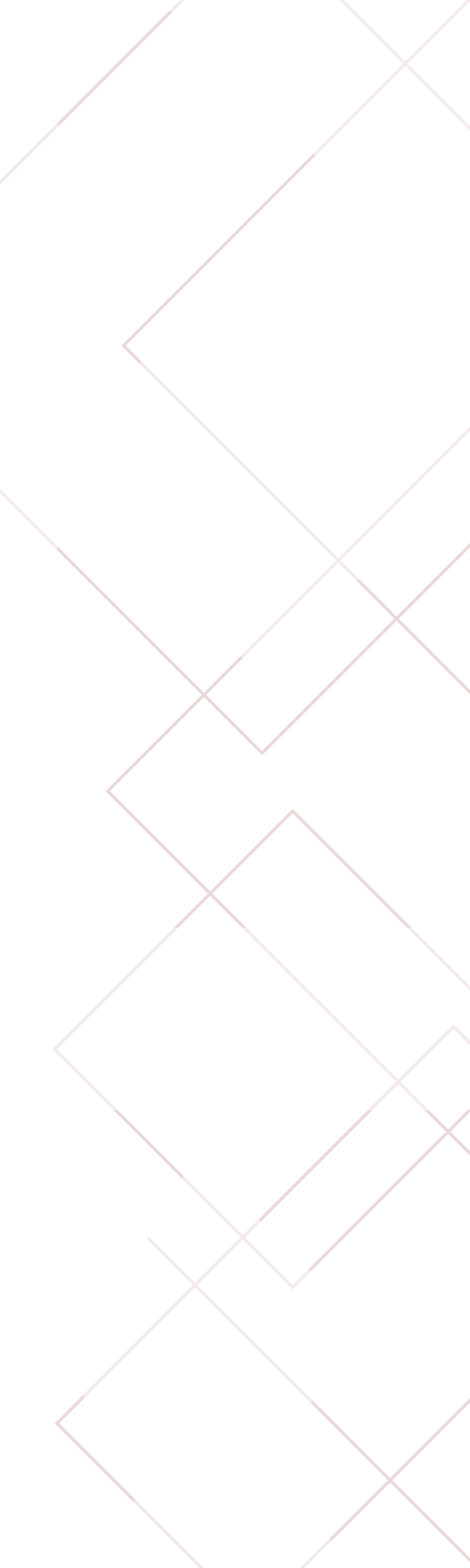

6 Nous considérons ici "sujets-actifs" les personnes qui prennent la parole, dans l'extrait choisi: Fabrícia et Fábia. 
diantes en master à l'Université de São Paulo. Fábia est plus expérimentée dans l'enseignement du FLE et en recherche étant donné son statut d'étudiante en doctorat. Les trois ont une relation d'amitié développée au sein d'un groupe de recherche de l'université, coordonné par leur directrice de recherche. Leur directrice de recherche leur a proposé l'idée de faire une IS pour les recherches de master de Fabrícia et Melissa. Elle a également invité Fabia, qui a une expérience professionnelle importante afin qu'elle puisse être le sosie.

L'énonciateur de cet extrait de l'IS est Fabrícia, professeure qui travaille dans une école privée de FLE depuis environ un an (au moment de l'IS) et qui a quelques mois d'expérience de travail comme professeure. Elle est aussi étudiante de master à l'USP. Avec sa recherche elle a pour objectif de comprendre son propre travail, le travail de l'enseignant de FLE. Elle a deux destinataires au moment de sa prise de parole : Fábia et Melissa. Nous pouvons confirmer que Fábia est le destinataire direct de l'énonciateur « ... tu... tu dis 'oui' » (ligne $46 \mathrm{~F})^{7}$ et Melissa le destinataire indirect (elle observe l'IS).

Fábia, le destinataire direct de Fabrícia, est le sosie de l'IS et lui pose la question (qui ne fait pas partie de l'extrait de cette analyse) : "Alors, Fabrícia, on va imaginer la situation suivante : demain je vais te remplacer dans ton travail et tu vas me dire tout ce que je dois faire pour que personne se rend compte que c'est moi, oui? ». Fabrícia a pour but de lui donner le maximum d'informations sur son travail puisqu'elle est la seule personne capable de lui donner des renseignements précis sur son activité, alors que Fábia doit faire attention aux instructions de Fabrícia et lui poser le maximum de questions pour comprendre ce qu'il audra faire pour qu'elle remplace bien Fabrícia le lendemain.

Le deuxième destinataire présent au moment de l'IS est Melissa, le destinataire indirect, qui observe l'IS. Melissa ne prend pas la parole pendant cette partie de l' IS analysée ${ }^{8}$.

De plus, on peut penser que l'énonciateur peut avoir aussi comme destinataires d'autres personnes qui ne sont pas présentes au moment de l'IS, comme par exemple : la directrice de recherche, qui oriente les travaux académiques des trois participantes de l'IS, ceux qui s’intéressent aux questions liées à ce type de recherche, etc.

Après la compréhension du contexte de production du texte, nous analyserons l'IS à partir du point de vue du premier niveau d'analyse du texte : l'infrastructure globale, comprenant le plan global du texte, les types de discours et les séquences. Le plan global du texte correspond à l'organisation des contenus, comme une sorte de « résu-
7 Les références entre parenthèses sont faites aux lignes de la transcription de l'extrait.

$8 \mathrm{Vu}$ que Melissa ne prend pas la parole l'extrait de l'IS analysé ici, on ne va pas beaucoup s'y centrer puisque ce ne sont pas des informations fondamentales pour cette analyse. 
mé » du texte. Le premier contenu abordé de cet extrait de l'IS est la question de l'existence d'une « fiche préétablie » que les professeurs de l'école doivent suivre pendant leurs cours. Selon Fabrícia, cette fiche est préparée en réunion par tous les professeurs, mais c'est la coordinatrice qui a la décision finale sur le contenu de cette fiche.

Ensuite, à partir d'une question du sosie sur la possibilité que la directrice accepte des propositions et des suggestions des professeurs, Fabrícia parle d'une gêne existante dans son travail : « je déteste... les cassettes »; « «e ne sais pas travailler avec la cassette » (lignes 10F ; 12F).

Le prochain thème abordé est celui qui concerne le consensus établi parmi les professeurs de ne pas discuter avec leur coordinatrice pendant la réunion pour ne pas la prolonger. Dans cette partie, Fabrícia reprend les mots utilisés par le professeur à qui elle parlait au moment où elle a connu ce consensus :

28F : [oui... elle ne va pas savoir...( ) donc... en fait... une chose... éh... une fois... éh... pendant une réunion une professeure a dit... éh :: «tu ne discutes pas avec la :: directrice...

29S : [uhn uhn

30F : dans la classe tu fais ce que tu veux »

(lignes 28F, 29S et 30F).

Le sujet de la discussion suivante est la question de la surveillance dans le milieu de travail. Fabrícia dit ne pas savoir s'il y a quelqu'un qui la surveille pendant ses cours, mais elle a déjà entendu parler des choses à propos de cette problématique.

Ensuite, Fabrícia dit au sosie qu'il doit mentir à la directrice et aussi dans les dossiers de classe (où elle enregistre ce qui a été travaillé dans les cours). Elle dit que le sosie doit mentir s'il n'utilise pas ou utilise quelque chose de différene de la fiche préétablie en réunion par la coordinatrice.

Il y a une reprise de la question du consensus parmi les professeurs : le sosie y insiste et demande à Fabrícia s'il peut discuter avec la directrice des changements des fiches préétablies pendant la réunion.

70S : ... alors je ne peux pas essayer de changer des choses ....

71F: [oui

$72 \mathrm{~S}$ : et les prof ne veulent pas:: changer de choses aussi

73F: ... ils ont déjà essayé de:: de le faire... 


\begin{tabular}{|l|}
\hline $74 \mathrm{~S}:$ \\
\hline $\begin{array}{l}\text { 75F }: \text { donc... c'est un conseil « ça ne change pas... donc... re/ reste en } \\
\text { silence... on termine la réunion plus tôt... et tu décides:: }\end{array}$ \\
\hline 76S : $\quad$ [uhn uhn... \\
\hline $77 \mathrm{~F}:$ dans la salle... ce que tu vas faire» \\
\hline
\end{tabular}

(lignes 70 S à 77F)

Ainsi nous pouvons partager l'extrait en question en six thématiques qui le résument:

(1S - 9S) La fiche préétablie en réunion avec tous les professeurs, mais avec la décision finale de la directrice ;

(10S - 26F) La difficulté de Fabrícia de se servir de la cassette qui doit être utilisée en salle de classe (selon les recommandations de la coordinatrice);

(27S - 34F) Le consensus parmi les professeurs de ne pas discuter avec la coordinatrice en réunion;

(35S - 45S) La question de la surveillance dans le contexte de travail de Fabrícia;

(46F - 58F) La question de mentir sur le dossier d'enregistrement de cours et à la coordinatrice;

(59S - 79F) Reprise du consensus des professeurs.

Le texte étudié ici appartient au genre de texte ${ }^{9}$ entretien oral : il a été enregistré, puis nous l'avons transcrit pour l'analyser. Ce genre est articulé dans une situation d'action langagière impliquée par les interlocuteurs qui alternent la parole dans un espace et un temps commun (Bronckart, 2003). Ainsi, à propos du type de discours dans le texte, il y a une prévalence du discours interactif, c'est-à-dire : du monde de l'exposé impliqué.

Dans ce texte, le discours interactif est le «primaire » (Bronckart, 2003) : la parole est directement prise par les interlocuteurs caractérisés par des marques de références (je, tu, on, nous) et leurs formes verbales.

On a constaté aussi une forte présence du récit interactif - où le monde de la narration est impliqué - lorsque Fabrícia, l'instructrice, fait des références aux situations de son milieu du travail situées au passé.

9 On adopte ici la notion de genre de texte de Bronckart (2003). 
28F:

[oui... elle ne va pas savoir...( ) donc... en fait... une chose... éh... une fois... éh... pendant une réunion une professeure a dit... éh :: « tu ne discutes pas avec la :: directrice...

(ligne 28F)

Au deuxième niveau d'analyse du texte, nous avons les mécanismes de textualisation impliquant la cohésion verbale, la cohésion nominale et la connexion. Par rapport à la connexion, nous avons trouvé de nombreux éléments de liaisons simples et complexes dont la fonction est de connecter les phases d'une séquence : et, mais, c'est-à-dire, donc etc.

3S : c'est la directrice qui a organisé cette fiche?

4F: oui

5S : oui?

6F: en réunion avec nous mais... c’est elle qui décide

7S : c'est elle qui décide?

8F: oui

À propos de la cohésion nominale, nous avons remarqué l'utilisation de pronoms personnels qui représentent les interlocuteurs - l'instructrice et le sosie (je, tu) ; le groupe de professeurs de l'école et l'instructrice (on, nous) «nous avons un dossier », le groupe de professeur de l'école (ils) « ils ont déjà essayé de le faire » et la coordinatrice (elle).

En ce qui concerne la cohésion verbale, nous avons observé la plupart des verbes au présent, utilisés souvent par les deux interlocuteurs, surtout pendant les explications et les questions par rapport aux activités du travail de Fabrícia. Par ailleurs, le texte présente aussi l'usage du passé et du futur. Le passé composé est utilisé notamment par Fabrícia pour raconter au sosie des situations qui se sont déjà passées à l'école. Le futur est employé par les interlocuteurs :

9S : et si je propose des choses-là :: elle va accepter ou non ? ou j’aurai des problèmes?

67F : les autres professeurs vont ::: te regarder... parce que sinon.... éh... la réunion ne termine pas ((rires))... oui

(lignes 9S et 67F)

Quant aux mécanismes énonciatifs, nous avons trouvé des 
modalisations appréciatives « je déteste les cassettes »; " elle aime travailler avec quelques exercices... » (lignes 10F ; 12F). De plus, on a constaté aussi la forte présence des modalisations déontiques, qui représentent le domaine du droit, de l'obligation sociale. Elles sont surtout présentes dans les questions et réactions du sosie : « je dois me conformer », « et je peux toujours réessayer ... ? ». Toujours par rapport aux mécanismes énonciatifs, on constate la présence d'une voix qui représente le groupe de professeurs de l'école de l'instructrice :

75F : donc... c'est un conseil « ça ne change pas... donc... re/ reste en silence... on termine la réunion plus tôt... et tu décides::

76S : $\quad$ [uhn uhn...

77F : dans la salle... ce que tu vas faire»

(lignes 75F, 76S e 77F).

À partir de l'analyse des éléments linguistiques de l'extrait étudié, il est possible de voir les représentations qui y sont construites à propos des orientations pour le travail du professeur et leur suivi. Vu que l'activité « ne se touche pas du doigt », c'est à travers les textes, des «produits empiriques des actions langagières » (Bronckart, 2003) qu'on y a accès. D'où l'importance de l'analyse linguistique des textes d'Instruction au Sosie.

Le travail prescrit nous aide à connaître - avec le travail réalisé le réel de l'activité du professeur et c'est cela qui nous intéresse, comme on l'a déjà mentionné. Dans la partie analysée de l'instruction au sosie, on peut voir quelques orientations existantes dans le travail de Fabrícia. À partir de l'analyse du plan global du texte, il est possible de connaître un peu de son travail prescrit : la fiche préétablie en réunion organisée par la coordinatrice et l'utilisation des cassettes en salle de classe.

La fiche préétablie en réunion correspond au schéma du cours qui sera donné par les professeurs ; ce n'est pas à eux de décider la façon dont ils travailleront, l'ordre des exercices qui seront faits, les activités supplémentaire, les échanges oraux avec les élèves : tout ce qui concerne la réalisation des cours est décidé par la coordinatrice. 
3S : c'est la directrice qui a organisé cette fiche?

4F: oui

5S : oui?

6F: en réunion avec nous mais... c'est elle qui décide

7S : c’est elle qui décide?

8F: oui

L’analyse des mécanismes de textualisation nous a indiqué l'élément de connexion «mais » (présent dans la ligne 6F de l'exemple précédent) qui nous montre un conflit : cela ne sert à rien - pour Fabricia - d'organiser la fiche en réunion, vu que toutes les décisions sont prises par la coordinatrice. Fabrícia dit que des suggestions peuvent être faites, mais ce n'est pas sûr qu'elle, la coordinatrice, les acceptera, bien au contraire, vu que les autres professeurs qui travaillent à l'école lui ont déjà suggéré de changer la vidéo dont Fabrícia parle - la vidéo sur cassette "Victor » (ligne 18F) - et même en sachant que les professeurs n'étaient pas très à l'aise de travailler avec ce support-là, elle ne l'a pas changé, un indice de son autoritarisme face aux professeurs.

Fabrícia n'aime pas cette vidéo, ce que l'on peut voir à partir de la modalisation appréciative négative « je déteste » : cela lui apporte de la souffrance dans son travail. Par la manière dont elle s'exprime, il est possible de voir que cette situation lui apporte peut-être des difficultés : « je sais pas :: travailler avec la cassette» (12F). Fabrícia affirme qu'elle n'aime pas et qu'elle ne sait pas travailler avec la cassette : le travail prescrit, dans ce cas-là, ne lui apporte pas de «confort » dans son travail dans le sens où il est plus intéressant pour les professeurs de faire des activités agréables en classe - surtout celles auxquelles ils croient vraiment- et ce n'est pas cela qui se passe.

De plus, nous avons observé une opposition entre la modalisation appréciative négative évoquée par Fabrícia et la modalisation appréciative positive liée à la coordinatrice : «je déteste les cassettes » (ligne 10F) versus "elle aime travailler avec quelques exercices... » (ligne 12F). Cette opposition peut confirmer le conflit vécu par Fabrícia dans son contexte de travail. Les modalisations détester versus aimer peuvent indiquer un empêchement de la réalisation de l'activité de « re-conception » (AMIGUES, 2009) du travail prescrit. Selon Amigues (2009), l'activité de re-conception est la tâche que le travailleur donne à lui-même comme réponse aux prescriptions de son travail. La professeure est devant une prescription qui ne lui apporte pas de 
confort dans son travail mais qu'elle a besoin de suivre parce que sa coordinatrice l'aime. Donc le travail prescrit est difficile d'être réorganisé par Fabrícia parce qu'elle doit faire ce qui est exactement prescrit.

Les orientations pour le travail de Fabrícia sont très strictes : la coordinatrice n'accepte pas de les changer et les professeurs n'ont pas le droit de décider ; les accepter c'est encore une autre contrainte (se mettre d'accord avec la situation). C'est ce que nous montre la modalisation déontique du sosie suivie d'une confirmation de l'instructrice:

68S : c'est-à-dire... je dois me conformer....

69F:

[oui

L'analyse du discours dans le texte révèle une forte présence du récit interactif - où le monde de la narration est impliqué, ça nous laisse clair que, pendant l'IS, le professeur revit des situations déjà vécues de son travail, ce qui lui permet d'avoir un autre regard sur son expérience professionnelle.

28F:

[oui...

elle ne va pas savoir...( ) donc... en fait... une chose... éh... une fois... éh... pendant une réunion une professeure a dit... éh :: «tu ne discutes pas avec la :: directrice...

29S :

[uhn uhn

30F : dans la classe tu fais ce que tu veux»

31S: AH... d'accord... il y a la fiche mais je peux faire une autre chose?

32F: oui

En même temps, l'analyse de la cohésion verbale nous présente les trois temps verbaux (passé composé, présent et futur proche) qui apparaissent dans le texte de l'IS. Ils montrent que le professeur revit l'expérience déjà vécue et à partir de la réflexion qui en naît, il est prêt pour en vivre d'autres et avoir un nouveau regard sur son activité professionnelle.

67F : les autres professeurs vont ::: te regarder... parce que sinon.... éh... la réunion ne termine pas ((rires))... oui 
73F: ... ils ont déjà essayé de:: de le faire...

$74 S$ :

[uhn uhn

$75 \mathrm{~F}$ :donc... c'est un conseil « ça ne change pas... donc... re/ reste en silence... on termine la réunion plus tôt... et tu décides::

76S: $\quad[$ uhn uhn...

$77 \mathrm{~F}$ : dans la salle... ce que tu vas faire »

Par l'analyse du plan global du texte, on vérifie que le sosie pose des questions à Fabrícia à propos des autres possibilités existantes de réaliser le travail à faire le lendemain sans que personne ne découvre le remplacement. Cette consigne, d'éviter « la découverte du remplacement ", sert à la réflexion de Fabrícia : en donnant les instructions au sosie, on attend qu'elle prenne conscience des autres façons possibles de faire son travail mais qu'elle ne choisit pas d'habitude ou peut-être jamais. Même si elles ont été mises à l'écart et n’ont pas été effectuées, ces autres manières de travailler font partie du travail réel et leur prise de conscience par Fabrícia fera en sorte qu'elle se développe en tant qu'individu et par conséquent, en tant qu'enseignante.

Quand le sosie demande à Fabrícia s'il peut utiliser en salle de classe d'autres activités que celles qui ont été préétablies en réunion par la coordinatrice (ligne 27S), la réponse de Fabrícia montre une autre possibilité de faire son travail : au lieu de faire les activités souhaitées par la coordinatrice avec lesquelles Fabrícia n'est pas d'accord, elle peut faire ce qu'elle veut et ne pas le dire à la coordinatrice parce qu'elle ne le saura pas : «oui... elle ne va pas savoir...( ) » (ligne 28F). À partir de cette autre possibilité de travail découverte et à partir de l'analyse des voix présentes dans l'IS, on prend connaissance d'un «conseil » que Fabrícia a reçu d'une collègue professeure: «tu ne discutes pas avec la :: directrice.../ dans la classe tu fais ce que tu veux » (lignes 28F et 30), c'est la voix d'une collègue qui représente le groupe de professeurs et qui effectue une activité de re-conception du travail prescrit.

L’enseignante qui lui a donné ce conseil semble être plus expérimentée que Fabrícia parce qu'elle a trouvé des stratégies pour éviter les problèmes existants dans son travail, comme par exemple le manque de liberté dans sa pratique en salle de classe, alors que Fabrícia insistait à discuter avec la coordinatrice des schémas des cours. On imagine que cette enseignante a déjà été à la place de Fabrícia et a déjà essayé de proposer d'autres idées en réunion sans succès. Voilà pourquoi elle a dit à Fabrícia qu'il n'est pas nécessaire d'en discuter en réunion, car dans 
la salle de cours, elle sera seule et pourra alors ne pas suivre le schéma proposé pour le cours. En fait, l'enseignante veut lui dire qu'elle n'a pas besoin de suivre la fiche à laquelle Fabrícia fait référence au début de l'extrait (ligne 1S), mais faire ce qu'elle juge être mieux, ce qu'elle veut, vu que la coordinatrice n'aura pas conscience de ce « changement », du non suivi de la fiche par les professeurs (ligne 28F).

Le conseil donné à Fabrícia par l'autre professeure peut nous indiquer l'existence d'un collectif dans leur milieu de travail où les professeurs ont des soucis similaires et partagent des stratégies pour surmonter les obstacles comme le manque de liberté, dans ce cas-là. On peut considérer que ce collectif est indiqué par l'emploi de la lère personne du pluriel, nous (lignes 6F, 38F, 50F, 52F) et d'une voix qui représente le groupe de professeurs de l'école $(75 \mathrm{~F}$ et $77 \mathrm{~F})$ où il n'y a pas de distinction entre les enseignants débutants et les expérimentés vu qu'ils ont les mêmes orientations de la coordinatrice.

Cependant, ce conseil peut être aussi considéré comme une prescription des professeurs plus expérimentés à l'école aux enseignants qui sont débutants, comme c'est le cas de Fabrícia. Comme les professeurs chevronnés savent déjà que c'est difficile de convaincre la coordinatrice à propos des changements et des suggestions, ils ont conseillé à Fabrícia de ne pas essayer de proposer ses idées à la coordinatrice pour que la réunion ne dure pas beaucoup de temps et termine plus tôt. Mais si c'était un conseil, Fabrícia aurait le droit de le suivre ou non selon son envie ; elle pourrait insister pour discuter de ses propositions en réunion si elle le voulait. Pourtant, si Fabrícia ne suit pas ce « conseil » et continue la discussion avec la coordinatrice en lui proposant de nouvelles idées d'activités, par exemple, ses collègues ne seront pas contents, ce qui lui apportera de l'inconfort et la mettra dans une situation désagréable dans son travail : « les autres professeurs vont ::: te regarder... » (ligne 67). Finalement, ce qui soutient cette hypothèse est le passage de l'extrait où le sosie confirme à Fabrícia qu'il ne peut pas « essayer de changer des choses .... » (ligne 70S) et Fabrícia lui répond « oui » (ligne 71). On croit que cette réponse a le sens de confirmation, de "oui, c'est ça ", ce qui confirme que le sosie ne peut pas proposer des choses en réunion, doit forcément suivre le conseil qui est, en fait, une prescription des collègues plus expérimentés.

Par la suite, Fabrícia remarque que, si le sosie décide de ne pas suivre la fiche et faire d'autres activités non prévues à sa place, il ne faut pas du tout que la coordinatrice en prenne conscience : «mais elle ne peut pas savoir... elle ne peut pas savoir » (ligne 34F). Bien qu'il y ait un dossier où il faut que les professeurs notent tout ce qui a été vraiment 
travaillé en classe, si jamais le professeur n’a pas réalisé une telle activité prévue sur la fiche, il doit mentir sur ce dossier-là (lignes 53S et 54F).

Fabrícia ne sait pas si la coordinatrice vérifie si le dossier correspond aux fiches et aux cours donnés (ligne 58F), ni s'il existe à l'école un type de surveillance qui pourrait la punir du non suivi de la fiche et des activités proposées. Même si elle en a déjà entendu des commentaires (ligne 36F), elle ne peut pas l'affirmer.

À partir des analyses et des considérations qui viennent d'être faites, il nous est possible de proposer quelques conclusions à propos des orientations pour le travail du professeur qu'on a pu remarquer dans l'extrait étudié dans cet article.

\section{Considérations finales}

Pour un enseignant, on sait qu'il faut que les prescriptions soient suivies par le professeur vu que c'est son institution qui les a élaborées et souvent la menace d'un renvoi est pesante si celles-ci ne sont pas accomplies par le professionnel. Cependant, les professeurs peuvent ne pas être d'accord avec elles et, toujours en cherchant à bien réaliser leur travail, ils trouvent des stratégies pour faire ce qu'il faut faire mais d'une façon qui soit intéressante pour eux.

À partir de l'extrait analysé dans cette investigation, on a connu quelques prescriptions existantes dans le travail de Fabrícia, comme par exexmple : il est essentiel que Fabrícia et ses collègues fassent leurs cours selon le schéma de cours établi par la coordinatrice en réunion et utilisent, par exemple, des supports matériaux démodés comme la cassette « Victor » que, selon Fabrícia, la coordinatrice aime bien. N'étant pas d'accord avec cela et en ayant vu que proposer des idées à la coordinatrice n'avait pas de résultats significatifs, les autres enseignants ont décidé de faire ce qu'ils jugent être mieux dans leurs salles de classe, une attitude « cachée » de la coordinatrice. On imagine à partir des traces identifiées dans les textes qu'elle n'accepterait pas cette « désobéissance ».

Fabrícia, enseignante débutante, ne connaissait pas cette « astuce » jusqu'au moment où une collègue lui dit qu'il ne faut pas du tout discuter avec la coordinatrice, car elle ne changera pas d'avis. Ce conseil donné par la collègue peut représenter le collectif de travail qui s'aide mais aussi une autre prescription, pas de même valeur « d'autorité » que celles de la coordinatrice, mais quand même quelque chose qui doit être accompli par Fabrícia pour qu'elle ait une bonne 
relation avec ses collègues. Ce qui permet à cette collègue, qui donne ce « conseil » à Fabrícia, d'agir ainsi c'est son expérience dans le milieu scolaire, contrairement à Fabrícia, professeure débutante.

De cette manière, dans le travail du professeur il existe des orientations qui doivent être suivies et qui sont données par plusieurs sphères. Selon Lousada (2006), le travail du professeur est déterminé par son dialogue avec plusieurs couches de prescriptions représentées par des voix de l'école, du collectif de travail, de la didactique, du métier. Dans le travail de Fabrícia il y a celles de l'institution représentée par la coordinatrice et celles des collègues chevronnés, aussi importantes pour la réalisation du travail. C'est l'expérience qui permet aux professionnels plus expérimentés de donner des orientations aux débutants pour qu'ils travaillent de la façon dont l'enseignant expérimenté juge la meilleure. Par rapport au suivi des orientations, nous avons vu que cela n'est pas forcement fait si le professeur ne croit pas aux activités qu'on lui dit de faire (le travail prescrit) et s'il trouve des stratégies ou même des astuces pour ne pas le faire sans avoir des préjudices de la part de l'institution (surtout).

Avec l'investigation développée ici on a pu accéder à une partie du travail réel de Fabrícia : on a connu ce qu'elle fait d'habitude dans son travail et les autres façons de faire son travail aussi par rapport aux orientations existantes et leur suivi. Cette connaissance nous permet de comprendre le rôle des orientations dans le travail du professeur et permet, à Fabrícia, de réfléchir à sa pratique et avoir du développement en tant qu'individu et professeure.

À partir de notre étude il y a d'autres questions qui en sont nées et qui méritent d'être répondues, comme les rapports existants entre le collectif de travail et les prescriptions ; les orientations qui sont données par le collectif à lui-même et aussi celles qui sont données par les enseignants chevronnés aux débutants. On espère, avec notre article, avoir contribué à l'élargissement des études du travail enseignant et à celles à propos des orientations existantes dans le travail du professeur.

\section{RÉFÉRENCES BIBLIOGRAPHIQUES}

AMIGUES, René. L'enseignement comme travail. In: P. Bressoux (Ed.). Les stratégies d'enseignement en situation d'interaction. (pp. 243-262). 
Note de synthèse pour Cognitique : Programme Ecole et Sciences Cognitives. 2002.

. Trabalho do professor e trabalho de ensino. In: MACHADO, Anna Rachel. (Org.). 0 ensino como trabalho: uma abordagem discursiva. Londrina: EDUEL, 2004.

Pour une approche ergonomique de l'activité enseignante. Skholê, hors-série. 2003. http://sites.univ-provence.fr/ergo$\log$ /Bibliotheque/Divers/Amigues_approche_ergonomique.pdf. Data de acesso: 12/01/2014.

. Les situations professionnelles dans les sciences de l'éducation : objet scientifique spécifique ou objet-frontière ? Phronesis, v.1, n.1, pp. 118-123, Jan 2012.

AMIGUES, René ; FÉLIX, Christine ; ESPINASSY, Laurence. Observer le travail enseignant. Recherche en Éducation, 2014 (article à paraître).

BRONCKART, Jean-Paul. Atividade de Linguagem, Textos e Discurso: por um interacionismo sócio-discursivo. Trad. Anna Raquel Machado, Péricles Cunha. $1^{\mathrm{a}}$ edição. São Paulo: EDUC, 2003.

CLOT, Yves. Méthodologie en clinique de l'activité : l'exemple du sosie. In : DELEFOSSE, Marie Santiago ; ROUAN, Georges (org.) Les méthodes qualitatives en psychologie. Paris : Dunod, 2001.

A função psicológica do trabalho. Tradução de Adail Sobral. Petrópolis: Vozes, 2006.

FAÏTA, Daniel. Gêneros do discurso, gêneros de atividade, análise da atividade do professor. In: MACHADO, Anna Rachel. (Org) 0 ensino como trabalho: uma abordagem discursiva. Londrina: EDUEL, 2004 .

LOUSADA, Eliane Gouvêa. Entre o trabalho prescrito e o realizado: um espaço para a emergência do trabalho real do professor. Tese (Doutorado em Linguística Aplicada e Estudos da Linguagem) - Pontifícia Universidade Católica de São Paulo, São Paulo, 2006.

. Aprendendo o "métier" de professor: uma análise de textos produzidos em situação de formação inicial de professores de francês. In: Szundy, P. C.; Araújo, J. C.; Nicolaides, C. S.; Silva, K. A. (orgs). Linguística Aplicada e Sociedade: Ensino e Aprendizagem de Línguas no Contexto Brasileiro. Campinas: Pontes Editores, 2011. 
MACHADO, Anna Rachel. Revisando o conceito de resumos. In: DIONISIO, Angela Paiva; MACHADO, Anna Rachel; BEZERRA, Maria Auxiliadora. Gêneros textuais \& Ensino. $4^{\text {a }}$ edição. Rio de Janeiro: Editora Lucerna, 2002.

. (Org) $\mathbf{O}$ ensino como trabalho: uma abordagem discursiva. Londrina: EDUEL, 2004.

MACHADO, Anna Rachel; LOUSADA, Eliane Gouvêa; FERREIRA, Anise D'Orange. $O$ professor e seu trabalho: a linguagem revelando as práticas docentes. Campinas: Mercado de Letras, 2011.

PRETI, Dino. Análise de textos orais. $4^{\mathrm{a}}$ edição. São Paulo: Humanitas Publicações FFLCH/USP, 1999.

RIA, Luc. Analyse de l'activité professionnelle selon une approche ergonomique: une entrée par l'activité réelle des stagiaires en contexte de formation. Clermont Ferrand, avril 2005 (texte communiqué).

SAUJAT, Frédéric. Comment les enseignants débutants entrent dans le métier. In: Formation et pratiques d'enseignement en questions. n.1, 2004.

VIGOTSKI, Lev Semyonovich. A formação social da mente. $1^{\text {a }}$ Edição. São Paulo: Martins Fontes, 1984.

A construção do pensamento e da lin-

guagem. São Paulo: Martins Fontes, 2009.

Annexes - Transcription de l'extrait du texte d'instruction de l'Instruction au Sosie (00:21:12 - 00:26:09)

$\mathrm{F}=$ Fabrícia

$\mathrm{S}=$ Sosie

1S : ah d'accord... uhn uhn... toujours en suivant la fiche :: préétablie?

2F: oui

3S : c'est la directrice qui a organisé cette fiche?

4F: oui

5S : oui? 
6F: en réunion avec nous mais... c'est elle qui décide

7S : c'est elle qui décide?

8F: oui

9S : et si je propose des choses-là :: elle va accepter ou non ? ou j’aurai des problèmes?

10F: ça dépend... éh :: bon... il y a quelq/... par exemple... elle travaille :: avec -- -- je déteste les fites cassettes/ les cassettes 11S :

[uhn uhn

12F: elle aime travailler avec quelques exercices :: avec la cassette et je sais pas :: travailler avec la cassette

13S : uhn uhn

14F: [oui...donc on a déjà proposé de... adapter... de changer... de chercher un/ une vidéo de... youtube

15S : uhn uhn

16F: mais...

17S : [mais c'est... c'est avec des musiques ou c'est quoi ?... enregistré sur cette cassette?

18F: cassette? c'est... c'est une méthode qui s'appelle Victor --- je pense -- -- il y a une personne qui dit «ah Victor, on va apprendre le français:: » donc... ( )

19S :

[ce

sont des dialogues? ce sont des dialogues?

20F: oui

21S : et je ne peux pas au moins transformer cette cassette-là en pen drive -- -- je ne sais pas -- -- ou d'autre chose, non ?... je ne peux pas prendre ça et faire une copie ?... pour être un plus facile au moins de:.... travailler avec le truc....?

22F: je ne sais pas :: ce qui se passe... ((rires))

23S : non ?

24F: non... mais ::... mais même si:: si on passe... éh... par le cd, dvd -- -- je ne sais pas -- c'est la même chose

25S : uhn uhn... uhn uhn 
26F: ((rires)) c'est la même chose... le contenu :: ne change pas

$27 \mathrm{~S}$ : et si je présente... et si cela est prévu pour mon travail d'utiliser ce truc-là... et je trouve un autre :: ... un autre enregistrement... et j'emmène en classe ...(oui)... et je n'utilise pas le truc là... ça va me poser des problèmes ? qui :: .... d'abord qui va savoir que je fais ça?

28F:

[oui...

elle ne va pas savoir...( ) donc... en fait... une chose... éh... une fois... éh... pendant une réunion une professeure a dit... éh :: «tu ne discutes pas avec la :: directrice...

29S :

[uhn uhn

30F : dans la classe tu fais ce que tu veux »

31S: AH... d'accord... il y a la fiche mais je peux faire une autre chose?

32F: oui

33S : mais je ne veux pas utilis/

34F: [mais elle ne peut pas savoir... elle ne peut pas savoir 35S : ahn... mais elle surveille ce que je/... alors... je/ je suis là en classe... il y aura quelqu'un qui me surveille?

36F: ...( )... je ne sais pas... j'ai déjà entendu quelques fois qu'elle pas-se devant la por-te et elle écoute...

37S : [uhn uhn... uhn uhn

38F: elles nous écoute... je ne sais pas si c'est vrai... ((rires))

39S : ah oui... tu... tu n’a jamais vu ça?

40F: non

41S : alors... peut-être que moi, j'aurais de la chance qu'elle ne sera pas là et je peux utiliser une chose que pas la cassette

42F: oui :: ...oui

43S : je peux essayer toujours?

44F: oui

45S : c'est possible?

46F: [et si elle demande « tu as... tu as travaillé avec la 
cassette $? »$... tu... tu dis « oui » ((rires))

$47 \mathrm{~S}$ : je dis « oui » et c'est tout? point final ?

48F: $\quad$ [oui

49S : je dois enregistrer/ faire un registre de ma classe après?

50F: oui ( ) ... éh :: ... nous avons une fiche -- -- comment on dit ? -- -- un dossier...

51S :

[uhn...

$52 \mathrm{~F}$ : avec chaque classe :: nous avons un dossier... donc... on doit noter :: tout ce qu'on a travaillé

53S : alors si j'ai utilisé un truc que je ne devais pas utiliser... je dois mentir sur... ma/ ((rires)) mon dossier.... ((rires))

54F: $\quad$ oui ((rires))

$55 \mathrm{~S}$ : et dire que j’ai utilisé l'autre?

56F: oui

57S : d'accord... ahn?

58F: $\quad$ [c'est ça.... mais je pense qu'elle n'a pas de temps ::... pour vérifier... toutes les fiches...

59S : [uhn uhn ... et je peux toujours reessayer dans une prochaine réunion de parler avec elle d'essayer de changer des choses?

60F: non... parce qu'elle va aurgumenter/ aurgum ?/

61S : argumenter?

62F : argumenter... donc...

$63 S$ : mais je peux essayer de contre-argumenter

64F: ((rires))

65F: oui... mais

66S : [c'est possi/

$67 \mathrm{~F}$ : les autres professeurs vont ::: te regarder... parce que sinon.... éh... la réunion ne termine pas ((rires))... oui

$68 \mathrm{~S}$ : c'est-à-dire... je dois me conformer.... 
69F:

[oui

70S :... alors je ne peux pas essayer de changer des choses ....

71F:

[oui

$72 \mathrm{~S}$ : et les prof ne veulent pas:: changer des choses aussi

73F: ... ils ont déjà essayé de:: de le faire...

$74 S$ :

[uhn uhn

$75 \mathrm{~F}$ :donc... c'est un conseil « ça ne change pas... donc... re/ reste en silence... on termine la réunion plus tôt... et tu décides::

76S :

[uhn uhn...

77F : dans la salle... ce que tu vas faire»

$78 \mathrm{~S}$ : mais après je dois mentir sur mon dossier

79F: oui... c'est ça ((rires)) 